\title{
A least-squares formulation for the approximation of null controls for the Stokes system
}

\author{
Arnaud Münch ${ }^{\mathrm{a}}$, Pablo Pedregal ${ }^{\mathrm{b}}$ \\ a Laboratoire de Mathématiques, Université Blaise Pascal (Clermont-Ferrand 2), UMR CNRS 6620, Campus des \\ Cézeaux, 63177 Aubière, France \\ b Departamento de Matemáticas, ETSI Industriales, Universidad de Castilla-La-Mancha, 13071 Ciudad Real, Spain. \\ Received $* * * * * ;$ accepted after revision +++++ \\ Presented by
}

\begin{abstract}
This note deals with the approximation of distributed null controls for the Stokes equation. The existence of $L^{2}$ controls have been obtained in [Fursikov \&S Imanuvilov, Controllability of Evolution Equations, 1996]) via Carleman type estimates. We introduce and analyze a least-squares formulation of the controllability problem, and we show that it allows the construction of convergent sequences of functions toward null controls for the Stokes system. To cite this article: A. Münch, Pablo Pedregal, C. R. Acad. Sci. Paris, Ser. I
\end{abstract}

\section{Résumé}

Cette note concerne l'approximation de contrôles exactes pour le système de Stokes. L'existence de contrôles $L^{2}$ a été obtenu dans (Fursikov $\&$ Imanuvilov, Controllability of Evolution Equations, 1996]) en utilisant des inégalités de type Carleman. On introduit et analyse une formulation type moindres carrés et on montre qu'elle permet la construction de suites convergentes de fonctions vers des contrôles à zéro du système de Stokes. Pour citer cet article : A. Münch, P. Pedregal, C. R. Acad. Sci. Paris, Ser. I.

\section{Version française abrégée}

Soient $\Omega$ un ouvert borné de $\mathbb{R}^{N}(N=2$ ou $N=3)$ de frontière $\partial \Omega$ Lipschitzienne, $\omega$ un sous-domaine non vide de $\Omega$ et soit $T$ un réel positif. On note $Q_{T}=\Omega \times(0, T), q_{T}=\omega \times(0, T), \Sigma_{T}=\partial \Omega \times(0, T)$ et on désigne par $\mathbf{n}=\mathbf{n}(x)$ le vecteur normal unitaire sortant à $\Omega$ en tout point $\mathbf{x} \in \partial \Omega$. $\mathbf{L}^{2}(\Omega)$ désigne l'espace de Hilbert des fonctions $\mathbf{v}=\left(v_{1}, \ldots, v_{N}\right)$ telles que $v_{i} \in L^{2}(\Omega)$ pour tout $i$, et de façon similaire pour les autres symboles et espaces écrits en gras.

Cette note relève de la théorie du contrôle des équations aux dérivées partielles (voir $[2,7]$ et leurs références) et concerne l'approximation de contrôles à zéro pour le système de Stokes défini sur $Q_{T}$ par :

$$
\left\{\begin{array}{l}
\mathbf{y}_{t}-\nu \Delta \mathbf{y}+\nabla \pi=\mathbf{f} 1_{\omega}, \quad \nabla \cdot \mathbf{y}=0 \quad \text { dans } Q_{T} \\
\mathbf{y}=\mathbf{0} \text { sur } \Sigma_{T}, \quad \mathbf{y}(\cdot, 0)=\mathbf{y}_{0} \quad \text { dans } \Omega .
\end{array}\right.
$$

$\mathbf{f}=\mathbf{f}(\mathbf{x}, t)$ désigne le contrôle agissant sur l'ouvert $\omega$ durant l'intervalle de temps $(0, T) .1_{\omega}$ représente la fonction indicatrice de $\omega$ et $\nu>0$. Introduisant les espaces

Email addresses: arnaud.munch@math.univ-bpclermont.fr (Arnaud Münch), pablo.pedregal@uclm.es (Pablo Pedregal). 


$$
\begin{aligned}
& \mathbf{H}=\left\{\varphi \in \mathbf{L}^{2}(\Omega): \nabla \cdot \varphi=0 \text { in } \Omega, \varphi \cdot \mathbf{n}=0 \text { on } \partial \Omega\right\}, \\
& \mathbf{V}=\left\{\varphi \in \mathbf{H}_{0}^{1}(\Omega): \nabla \cdot \varphi=0 \text { in } \Omega\right\}, \quad U=\left\{\psi \in L^{2}(\Omega): \int_{\Omega} \psi(\mathbf{x}) d \mathbf{x}=0\right\},
\end{aligned}
$$

rappelons que pour toute donnée initiale $\mathbf{y}_{0} \in \mathbf{H}, T>0$ et $\mathbf{f} \in \mathbf{L}^{2}\left(q_{T}\right)$, (1) possède une unique solution $(\mathbf{y}, \pi)$ telle que $\mathbf{y} \in C^{0}([0, T] ; \mathbf{H}) \cap L^{2}(0, T ; \mathbf{V}), \pi \in L^{2}(0, T ; U)$ (voir [12]). Le problème de la contrôlabilité à zéro de (1) au temps $T$ consiste alors pour tout $\mathbf{y}_{0} \in \mathbf{H}$ à déterminer un contrôle $\mathbf{f} \in \mathbf{L}^{2}\left(q_{T}\right)$ tel que la solution correspondante satisfasse l'égalité $\mathbf{y}(\cdot, T)=0$ dans $\mathbf{L}^{2}(\Omega)$. L'existence de tels contrôles, uniforme en les données initiales $\mathbf{y}_{0}$, a été démontré dans [5] en utilisant les inégalités de type Carleman (voir également [4]).

L'approximation de contrôles semble en revanche peu abordée. Dans le cadre de la contrôlabilité approchée, [6] utilise la théorie de la dualité convexe conduisant à la limite à des problèmes numériques mal posés. Cela est dû aux propriétés de régularisation en temps de l'opérateur de Stokes (voir [8] pour une analyse concernant l'équation de la chaleur).

Nous adaptons l'approche variationnelle de [11] et décrivons sans utiliser la dualité une méthode de type moindre carrés consistant à déterminer parmi toutes les fonctions $(\mathbf{y}, \pi, \mathbf{f})$ - ayant la régularité ci-dessus et vérifiant a priori les conditions aux limites (en particulier la condition finale de contrôlabilité pour y) une fonction vérifiant le système de Stokes. Cela est réalisé en introduisant la fonctionnelle positive $E$ (6) définie sur l'ensemble non vide $\mathcal{A}(5)$ qui mesure en terme d'une fonction correcteur $v$ - unique solution du problème elliptique $(7)$ - l'écart du triplet $(\mathbf{y}, \pi, \mathbf{f})$ à l'espace des solutions du système de Stokes. La propriété de continuation unique pour le système de Stokes (voir [3,5]) implique alors que $(\mathbf{y}, \pi)$ est une solution du système de Stokes controlée par la fonction $\mathbf{f} 1_{\omega} \in \mathbf{L}^{2}\left(q_{T}\right)$ si et seulement si le triplet $(\mathbf{y}, \pi, \mathbf{f})$ réalise l'infimum de $E$ (Proposition 2.1).

Enfin, la fonctionnelle $E$ admet en tout point de $\mathcal{A}$ une dérivée première pour toute direction de l'espace de Hilbert $\mathcal{A}_{0}$ (voir (9)) et est donnée par la formule (12). En utilisant la structure du noyau de la fonctionnelle $E$ et en appliquant le lemme abstrait 3.1, on montre que chaque suite minimisante pour $E$ convergent fortement vers une solution du problème extremal (défini de façon unique à partir du premier terme de la suite), et ce malgré la possible non coercivité de la fonctionnelle quadratique $E$ (voir Proposition 3.1).

Cette approche générale de type moindre-carrés où la condition de contrôlabilité est imposé a priori requiert uniquement la résolution de problèmes elliptiques bien posé sur $Q_{T}$ et est de fait parfaitement adaptée à des supports $\omega$ de controles variable avec le temps. Il est important de remarquer toutefois que les contrôles obtenus $\mathbf{f} \in \mathbf{L}^{2}\left(q_{T}\right)$ ne sont pas en particulier ceux de norme $\mathbf{L}^{2}$ minimale. Nous renvoyons à [10] pour des applications numériques.

\section{English version}

\section{Problem statement}

Let $\Omega \subset \mathbb{R}^{N}, N=2$ or $N=3$ be a bounded connected open set whose boundary $\partial \Omega$ is Lipschitz. Let $\omega \subset \Omega$ be a (small) nonempty open subset, and assume that $T>0$. We use the notation $Q_{T}=$ $\Omega \times(0, T), q_{T}=\omega \times(0, T)$, and $\Sigma_{T}=\partial \Omega \times(0, T)$ and we denote by $\mathbf{n}=\mathbf{n}(x)$ the outward unit normal to $\Omega$ at any point $\mathbf{x} \in \partial \Omega$. Bold letters and symbols denote vector-valued functions and spaces; for instance $\mathbf{L}^{2}(\Omega)$ is the Hilbert space of the functions $\mathbf{v}=\left(v_{1}, \ldots, v_{N}\right)$ with $v_{i} \in L^{2}(\Omega)$ for all $i$.

This note is concerned with the null controllability problem for the Stokes system

$$
\left\{\begin{array}{l}
\mathbf{y}_{t}-\nu \Delta \mathbf{y}+\nabla \pi=\mathbf{f} 1_{\omega}, \quad \nabla \cdot \mathbf{y}=0 \quad \text { in } Q_{T} \\
\mathbf{y}=\mathbf{0} \text { on } \Sigma_{T}, \quad \mathbf{y}(\cdot, 0)=\mathbf{y}_{0} \text { in } \Omega .
\end{array}\right.
$$

$\mathbf{f}=\mathbf{f}(\mathbf{x}, t)$ stands for the control, and is assumed to act on $\omega$ during the time interval $(0, T)$; the symbol $1_{\omega}$ stands for the characteristic function of $\omega$. We introduce on (2) the spaces $\mathbf{H}, \mathbf{V}$ and $U$ usual in the context of Stokes systems. Then, for any $\mathbf{y}_{0} \in \mathbf{H}, T>0$, and $\mathbf{f} \in \mathbf{L}^{2}\left(q_{T}\right)$, there exists exactly one solution $(\mathbf{y}, \pi)$ of $(3)$ with the following regularity : $\mathbf{y} \in C^{0}([0, T] ; \mathbf{H}) \cap L^{2}(0, T ; \mathbf{V}), \pi \in L^{2}(0, T ; U)$ (see [12]). Therefore, the null controllability problem for (3) at time $T$ is the following. For any $\mathbf{y}_{0} \in \mathbf{H}$, find $\mathbf{f} \in \mathbf{L}^{2}\left(q_{T}\right)$ such that the corresponding solution to (3) satisfies

$$
\mathbf{y}(\cdot, T)=\mathbf{0} \text { in } \Omega .
$$


The controllability properties of evolution PDEs have attracted a lot of work the last decades. Some relevant references are $[2,5,7]$. In particular, in the case of Stokes - and more generally the Navier-Stokes -, we mention the reference [4]. Specifically, the following result is proved in [5] through Carleman estimates.

Theorem 1 (Fursikov-Imanuvilov) The linear system (3) is null-controllable at any time $T>0$.

On the other hand, the (numerical) approximation of such controls for the Stokes system has received less attention. We mention the work [6] where the approximate controllability is addressed through the use of penalty arguments and duality. The numerical difficulties occurring when dual arguments are employed are related to the regularizing property of the system (we refer to [8] in the closely related context of the heat equation). Following [11], we present in this note a simple least-squares method to approximate null controls for (3). The main idea consists in setting up an error functional which measures the deviation of functions from being a solution of (3), and minimizing such error over the class of feasible functions that comply with initial, boundary and final conditions (in particular the condition (4)).

\section{A least square reformulation}

We define the non-empty space

$$
\begin{aligned}
& \mathcal{A}=\{(\mathbf{y}, \pi, \mathbf{f}) ; \mathbf{y} \in \mathbf{L}^{2}\left(0, T, \mathbf{H}_{0}^{1}(\Omega)\right), \mathbf{y}_{t} \in \mathbf{L}^{2}\left(0, T ; \mathbf{H}^{-1}(\Omega)\right), \\
&\left.\mathbf{y}(\cdot, 0)=\mathbf{y}_{0}, \mathbf{y}(\cdot, T)=\mathbf{0}, \pi \in L^{2}(0, T ; U), \mathbf{f} \in \mathbf{L}^{2}\left(q_{T}\right)\right\} .
\end{aligned}
$$

Note that these hypotheses on $\mathbf{y}$ imply that it belongs to $C\left([0, T], \mathbf{L}^{2}(\Omega)\right)$, and so the two equalities $\mathbf{y}(\cdot, 0)=\mathbf{y}_{0}, \mathbf{y}(\cdot, T)=\mathbf{0}$ in $\mathbf{L}^{2}(\Omega)$ are appropriate. Then, we define the functional $E: \mathcal{A} \rightarrow \mathbb{R}^{+}$by

$$
E(\mathbf{y}, \pi, \mathbf{f})=\frac{1}{2} \iint_{Q_{T}}\left(\left|\mathbf{v}_{t}\right|^{2}+|\nabla \mathbf{v}|^{2}+|\nabla \cdot \mathbf{y}|^{2}\right) d x d t
$$

where the corrector $\mathbf{v}$ is the unique solution in $\mathbf{H}^{1}\left(Q_{T}\right)$ of the (elliptic) boundary value problem

$$
\left\{\begin{array}{c}
-\mathbf{v}_{t t}-\Delta \mathbf{v}+\left(\mathbf{y}_{t}-\nu \Delta \mathbf{y}+\nabla \pi-\mathbf{f} 1_{\omega}\right)=0, \quad \text { in } Q_{T}, \\
\mathbf{v}=0 \text { on } \Sigma_{T}, \quad \mathbf{v}_{t}=0 \quad \text { on } \Omega \times\{0, T\} .
\end{array}\right.
$$

For any $(\mathbf{y}, \pi, \mathbf{f}) \in \mathcal{A}$, the term $\mathbf{y}_{t}-\nu \Delta \mathbf{y}+\nabla \pi-\mathbf{f} 1_{\omega}$ belongs to $\mathbf{L}^{2}\left(0, T ; \mathbf{H}^{-1}(\Omega)\right)$ so that the functional $E$ is well-defined in $\mathcal{A}$. We have the following result.

Proposition 2.1 $(\mathbf{y}, \pi)$ is a controlled solution of the Stokes system (3) by the control function $\mathbf{f} 1_{\omega} \in$ $\mathbf{L}^{2}\left(q_{T}\right)$ if and only if $(\mathbf{y}, \pi, \mathbf{f})$ is a solution of the extremal problem :

$$
\inf _{(\mathbf{y}, \pi, \mathbf{f}) \in \mathcal{A}} E(\mathbf{y}, \pi, \mathbf{f}) \text {. }
$$

Proof- From the controllability of the Stokes system (Theorem 1), this extremal problem is well-posed and the infimum, equal to zero, is reached by any controlled solution of the Stokes system. Conversely, we check that any minimizer for $E$ is a solution of the (controlled) Stokes system. Let $(\mathbf{Y}, \Pi, \mathbf{F}) \in \mathcal{A}_{0}$ be arbitrary where

$$
\begin{aligned}
& \mathcal{A}_{0}=\left\{(\mathbf{y}, \pi, \mathbf{f}) ; \mathbf{y} \in \mathbf{L}^{2}\left(0, T, \mathbf{H}_{0}^{1}(\Omega)\right), \mathbf{y}_{t} \in \mathbf{L}^{2}\left(0, T ; \mathbf{H}^{-1}(\Omega)\right)\right. \\
&\left.\mathbf{y}(\cdot, 0)=\mathbf{y}(\cdot, T)=\mathbf{0}, \pi \in L^{2}(0, T ; U), \mathbf{f} \in \mathbf{L}^{2}\left(q_{T}\right)\right\} .
\end{aligned}
$$

The first variation of $E$ at the point $(\mathbf{y}, \pi, \mathbf{f})$ in the admissible direction $(\mathbf{Y}, \Pi, \mathbf{F})$ exists, and is given by

$$
\left\langle E^{\prime}(\mathbf{y}, \pi, \mathbf{f}),(\mathbf{Y}, \Pi, \mathbf{F})\right\rangle=\iint_{Q_{T}}\left(\mathbf{v}_{t} \cdot \mathbf{V}_{t}+\nabla \mathbf{v} \cdot \nabla \mathbf{V}+(\nabla \cdot \mathbf{y})(\nabla \cdot \mathbf{Y})\right) d x d t
$$

where the corrector $\mathbf{V} \in \mathbf{H}^{1}\left(Q_{T}\right)$, associated with $(\mathbf{Y}, \Pi, \mathbf{F})$, is the unique solution of

$$
\left\{\begin{array}{l}
-\mathbf{V}_{t t}-\Delta \mathbf{V}+\left(\mathbf{Y}_{t}-\nu \Delta \mathbf{Y}+\nabla \Pi-\mathbf{F} 1_{\omega}\right)=0 \quad \text { in } Q_{T} \\
\mathbf{V}=0 \quad \text { on } \quad \Sigma_{T}, \quad \mathbf{V}_{t}=0 \quad \text { on } \Omega \times\{0, T\}
\end{array}\right.
$$


Multiplying the main equation of this system by $\mathbf{v}$ (recall that $\mathbf{v}$ is the corrector associated with the minimizer $(\mathbf{y}, \pi, \mathbf{f}))$, integrating by parts, and using the boundary conditions on $\mathbf{v}$ and $\mathbf{V}$, we get that,

$$
\begin{array}{r}
\left\langle E^{\prime}(\mathbf{y}, \pi, \mathbf{f}),(\mathbf{Y}, \Pi, \mathbf{F})\right\rangle=-\iint_{Q_{T}}\left(-\mathbf{Y} \cdot \mathbf{v}_{t}+\nu \nabla \mathbf{Y} \cdot \nabla \mathbf{v}-\Pi \nabla \cdot \mathbf{v}-\mathbf{F} \cdot \mathbf{v} 1_{\omega}\right) d x d t \\
+\iint_{Q_{T}}(\nabla \cdot \mathbf{y})(\nabla \cdot \mathbf{Y}) d x d t, \quad \forall(\mathbf{Y}, \Pi, \mathbf{F}) \in \mathcal{A}_{0},
\end{array}
$$

where we have used that

$$
-\int_{0}^{T}\left\langle\mathbf{Y}_{t}, \mathbf{v}\right\rangle_{H^{-1}(\Omega), H^{1}(\Omega)} d t=\iint_{Q_{T}} \mathbf{Y} \cdot \mathbf{v}_{t} d x d t-\int_{0}^{1}[\mathbf{Y} \cdot \mathbf{v}]_{0}^{T} d x=\iint_{Q_{T}} \mathbf{Y} \cdot \mathbf{v}_{t} d x d t,
$$

and that

$$
\int_{0}^{T}\langle\nabla \Pi, \mathbf{v}\rangle_{H^{-1}(\Omega), H^{1}(\Omega)} d t=-\iint_{Q_{T}} \Pi \nabla \cdot \mathbf{v} d x d t .
$$

Therefore if $(\mathbf{y}, \pi, \mathbf{f})$ minimizes $E$, the equality $\left\langle E^{\prime}(\mathbf{y}, \pi, \mathbf{f}),(\mathbf{Y}, \Pi, \mathbf{F})\right\rangle=0$ for all $(\mathbf{Y}, \Pi, \mathbf{F}) \in \mathcal{A}_{0}$ implies that the corrector $\mathbf{v}=\mathbf{v}(\mathbf{y}, \pi, \mathbf{f})$ solution of (7) satisfies the conditions

$$
\left\{\begin{array}{lr}
\mathbf{v}_{t}+\nu \Delta \mathbf{v}-\nabla(\nabla \cdot \mathbf{y})=0, \quad \nabla \cdot \mathbf{v}=0, & \text { in } Q_{T}, \\
\mathbf{v}=0, & \text { in } q_{T} .
\end{array}\right.
$$

But from the unique continuation property for the Stokes system (see [3]), it turns out that $\mathbf{v}=0$ in $Q_{T}$ and that $\nabla \cdot \mathbf{y}$ is a constant on $Q_{T}$. Eventually, the relation

$$
\left\langle E^{\prime}(\mathbf{y}, \pi, \mathbf{f}),(\mathbf{Y}, \Pi, \mathbf{F})\right\rangle=(\nabla \cdot \mathbf{y}) \iint_{Q_{T}} \nabla \cdot \mathbf{Y} d x d t=0, \quad \forall(\mathbf{Y}, \Pi, \mathbf{F}) \in \mathcal{A}_{0}
$$

then implies that this constant is zero. Consequently, if $(\mathbf{y}, \pi, \mathbf{f}) \in \mathcal{A}$ is a minimizer for $E$, then $\nabla \cdot \mathbf{y}=0$, and the corrector $\mathbf{v}$ is zero, so that $E(\mathbf{y}, \pi, \mathbf{f})=0$. Therefore, $(\mathbf{y}, \pi, \mathbf{f})$ solves $(3)$, and since $(\mathbf{y}, \pi, \mathbf{f}) \in \mathcal{A}$, the state $\mathbf{y}$ is controlled at time $T$ by the function $\mathbf{f}$ which acts as a control.

Remark 1 It is worthwhile to notice that the proof of this proposition only utilizes optimality of $(\mathbf{y}, \pi, \mathbf{f})$ and not its minimality. Therefore in the statement of the proposition, we could have written instead: $(\mathbf{y}, \pi)$ is a controlled solution of the Stokes system (3) by the control function $\mathbf{f} 1_{\omega} \in \mathbf{L}^{2}\left(q_{T}\right)$ if and only if $(\mathbf{y}, \pi, \mathbf{f})$ is a stationary point for the functional $E(\mathbf{y}, \pi, \mathbf{f})$ over $(\mathbf{y}, \pi, \mathbf{f}) \in \mathcal{A}$. This is relevant from the perspective of the numerical simulation for it guarantees that the numerical procedure based on a descent strategy cannot get stuck in local minima.

Remark 2 For any $(\mathbf{y}, \pi, \mathbf{f}) \in \mathcal{A}$,

$$
E(\mathbf{y}, \pi, \mathbf{f})=\frac{1}{2}\left\|\mathbf{y}_{t}-\nu \Delta \mathbf{y}+\nabla \pi-\mathbf{f} 1_{\omega}\right\|_{\mathbf{H}^{-1}\left(Q_{T}\right)}^{2}+\frac{1}{2}\|\nabla \cdot \mathbf{y}\|_{\mathbf{L}^{2}\left(Q_{T}\right)}^{2} .
$$

This justifies the least-squares terminology we have employed.

Remark 3 The quasi-incompressibility case is obtained in the same way. It suffices to add $\epsilon \pi$ (for any $\epsilon>0)$ to the divergence term in the functional $E$.

Remark 4 The approach allows to consider compact support control jointly in time and space. It suffices to replace the function $1_{\omega}$ in (3) by any compact support function in time and space.

Remark 5 A fortiori, the approach is well-adapted to address the direct problem: remove from $\mathcal{A}$ the condition (4), and fix the forcing term $\mathbf{f}$ (see [1]).

\section{Convergence of minimizing sequences for $E$}

Proposition 2.1 reduces the approximation of a null control for (3) to a minimization of the functional $E$ over the space $\mathcal{A}$. Preliminary, since $\mathcal{A}$ is not an Hilbert space, we consider for any $\mathbf{s}_{\mathbf{0}}:=\left(\mathbf{y}_{0}, \pi_{0}, \mathbf{f}_{0}\right) \in \mathcal{A}$ the equivalent problem : $\min _{(\mathbf{y}, \pi, \mathbf{f}) \in \mathcal{A}_{0}} E\left(\mathbf{s}_{\mathbf{0}}+(\mathbf{y}, \pi, \mathbf{f})\right)$. We note $E\left(\mathbf{s}_{\mathbf{0}}+(\mathbf{y}, \pi, \mathbf{f})\right)=E_{\mathbf{s}_{\mathbf{0}}}(\mathbf{y}, \pi, \mathbf{f})$. Then, we endow the Hilbert space $\mathcal{A}_{0}$ with its natural norm $\|\cdot\|_{\mathcal{A}_{0}}$ such that :

$$
\|\mathbf{y}, \pi, \mathbf{f}\|_{\mathcal{A}_{0}}^{2}:=\iint_{Q_{T}}\left(|\mathbf{y}|^{2}+|\nabla \mathbf{y}|^{2}\right) d x d t+\int_{0}^{T}\left\|\mathbf{y}_{t}(\cdot, t)\right\|_{\mathbf{H}^{-1}(\Omega)}^{2} d t+\iint_{Q_{T}}\left(|\mathbf{f}|^{2}+|\pi|^{2}\right) d x d t
$$


recalling that $\left\|\mathbf{y}_{t}\right\|_{\mathbf{H}^{-1}(\Omega)}=\|\mathbf{g}\|_{\mathbf{H}_{\mathbf{0}}^{1}(\Omega)}$ where $\mathbf{g} \in \mathbf{H}_{\mathbf{0}}^{\mathbf{1}}(\Omega)$ solves $-\Delta \mathbf{g}=\mathbf{y}_{\mathbf{t}}$ in $\Omega$. We denote $\langle,\rangle_{\mathcal{A}_{0}}$ the corresponding scalar product.

The relation (12) allows to define a minimizing sequence $\left(\mathbf{y}^{n}, \pi^{n}, \mathbf{f}^{n}\right)_{n \geq 0} \in \mathcal{A}_{0}$ for $E_{\mathbf{s}_{0}}$. Precisely, for any $\left(\mathbf{y}^{k}, \pi^{k}, \mathbf{f}^{k}\right) \in \mathcal{A}_{0}, k \geq 0$, we define $\left(\overline{\mathbf{y}}^{k}, \bar{\pi}^{k}, \overline{\mathbf{f}}^{k}\right) \in \mathcal{A}_{0}$ the unique solution of the formulation

$$
\left\langle\left(\overline{\mathbf{y}}^{k}, \bar{\pi}^{k}, \overline{\mathbf{f}}^{k}\right),(\mathbf{Y}, \Pi, \mathbf{F})\right\rangle_{\mathcal{A}_{0}}=\left\langle E_{\mathbf{s}_{\mathbf{0}}}^{\prime}\left(\mathbf{y}^{k}, \pi^{k}, \mathbf{f}^{k}\right),(\mathbf{Y}, \Pi, \mathbf{F})\right\rangle, \quad \forall(\mathbf{Y}, \Pi, \mathbf{F}) \in \mathcal{A}_{0}
$$

In particular, (15) implies that $\bar{\pi}^{k}=-\nabla \cdot \mathbf{v}^{k} \in L^{2}\left(Q_{T}\right)$ and $\overline{\mathbf{f}}^{k}=-\mathbf{v}^{k} 1_{\omega} \in \mathbf{L}^{2}\left(q_{T}\right)$ (actually in $\mathbf{H}^{1}\left(q_{T}\right)$ ). Therefore, $-\left(\overline{\mathbf{y}}^{k}, \bar{\pi}^{k}, \overline{\mathbf{f}}^{k}\right) \in \mathcal{A}_{0}$ is a descent direction for $E$. Finally, the triplet $\left(\mathbf{y}^{k+1}, \pi^{k+1}, \mathbf{f}^{k+1}\right) \in \mathcal{A}_{0}$ is defined by :

$$
\left(\mathbf{y}^{k+1}, \pi^{k+1}, \mathbf{f}^{k+1}\right)=\left(\mathbf{y}^{k}, \pi^{k}, \mathbf{f}^{k}\right)-\eta_{k}\left(\overline{\mathbf{y}}^{k}, \bar{\pi}^{k}, \overline{\mathbf{f}}^{k}\right), \quad k>0
$$

where $\eta_{k}$ denotes the optimal positive descent step given by $\eta_{k}=\left\|\overline{\mathbf{y}}^{k}, \bar{\pi}^{k}, \overline{\mathbf{f}}^{k}\right\|_{\mathcal{A}_{0}}^{2} E_{\mathbf{s}_{\mathbf{0}}}\left(\overline{\mathbf{y}}^{k}, \bar{\pi}^{k}, \overline{\mathbf{f}}^{k}\right)^{-1}$.

We refer to [9] where the conjugate gradient algorithm is detailed in the closed context of the heat equation.

One main issue of our variational approach is to establish the convergence of the minimizing sequence.

Proposition 3.1 For any $\mathbf{s}_{\mathbf{0}} \in \mathcal{A}$ and any $\left\{\mathbf{y}^{0}, \pi^{0}, \mathbf{f}^{0}\right\} \in \mathcal{A}_{0}$, the sequence $\mathbf{s}_{\mathbf{0}}+\left\{\left(\mathbf{y}^{\mathbf{n}}, \pi^{\mathbf{n}}, \mathbf{f}^{\mathbf{n}}\right)\right\}_{\mathbf{n}} \in \mathcal{A}$ converge strongly to a solution of the extremal problem (8).

This proposition is the consequence of the following abstract result which can be adapted to many different situations where this variational perspective can be of help.

Lemma 3.1 Suppose $\mathbf{T}: X \mapsto Y$ is a linear, continuous operator between Hilbert spaces, and $H \subset X, a$ closed subspace, $u_{0} \in X$. Put

$$
E: u_{0}+H \mapsto \mathbf{R}^{+}, E(u)=\frac{1}{2}\|\mathbf{T} u\|^{2}, \quad A=\operatorname{Ker} \mathbf{T} \cap H .
$$

(i) $E: u_{0}+A^{\perp} \rightarrow \mathbf{R}$ is quadratic, non-negative, and strictly convex, where $A^{\perp}$ is the orthogonal complement of $A$ in $H$.

(ii) If we regard $E$ as a functional defined on $H, E\left(u_{0}+\cdot\right)$, and identify $H$ with its dual, then the derivative $E^{\prime}\left(u_{0}+\cdot\right)$ always belongs to $A^{\perp}$. In particular, a typical steepest descent procedure for $E\left(u_{0}+\cdot\right)$ will always stay in the manifold $u_{0}+A^{\perp}$.

(iii) If, in addition, $\min _{u \in H} E\left(u_{0}+u\right)=0$, then the steepest descent scheme will always produce sequences converging (strongly in $X$ ) to a unique (in $u_{0}+A^{\perp}$ ) minimizer $u_{0}+\bar{u}$ with zero error.

Proof of Lemma 3.1- Suppose there are $u_{i} \in A^{\perp}, i=1,2$, such that

$$
E\left(u_{0}+\frac{1}{2} u_{1}+\frac{1}{2} u_{2}\right)=\frac{1}{2} E\left(u_{0}+u_{1}\right)+\frac{1}{2} E\left(u_{0}+u_{2}\right) .
$$

Due to the strict convexity of the norm in a Hilbert space, we deduce that this equality can only occur if $\mathbf{T} u_{1}=\mathbf{T} u_{2}$. So therefore $u_{1}-u_{2} \in A \cap A^{\perp}=\{0\}$, and $u_{1}=u_{2}$. For the second part, note that for arbitrary $U \in A, \mathbf{T} U=0$, and so

$$
E\left(u_{0}+u+U\right)=\frac{1}{2}\left\|\mathbf{T} u_{0}+\mathbf{T} u+\mathbf{T} U\right\|^{2}=\frac{1}{2}\left\|\mathbf{T} u_{0}+\mathbf{T} u\right\|^{2}=E\left(u_{0}+u\right) .
$$

Therefore the derivative $E^{\prime}\left(u_{0}+u\right)$, the steepest descent direction for $E$ at $u_{0}+u$, has to be orthogonal to all such $U \in A$.

Finally, assume $E\left(u_{0}+\bar{u}\right)=0$. It is clear that this minimizer is unique in $u_{0}+A^{\perp}$ (recall the strict convexity in (i)). This, in particular, implies that for arbitrary $u \in A^{\perp}$,

$$
\left\langle E^{\prime}\left(u_{0}+u\right), \bar{u}-u\right\rangle \leq 0,
$$

because this inner product is the derivative of the section $t \mapsto E\left(u_{0}+t \bar{u}+(1-t) u\right)$ at $t=0$, and this section must be a positive parabola with the minimum point at $t=1$. If we consider the gradient flow

$$
u^{\prime}(t)=-E^{\prime}\left(u_{0}+u(t)\right), \quad t \in[0,+\infty),
$$

then, because of (16),

$$
\frac{d}{d t}\left(\frac{1}{2}\|u(t)-\bar{u}\|^{2}\right)=\left\langle u(t)-\bar{u}, u^{\prime}(t)\right\rangle=\left\langle u(t)-\bar{u},-E^{\prime}\left(u_{0}+u(t)\right)\right\rangle \leq 0 .
$$


This implies that sequences produced through a steepest descent method will be minimizing for $E$, uniformly bounded in $X$ (because $\|u(t)-\bar{u}\|$ is a non-increasing function of $t$ ), and due to the strict convexity of $E$ restricted to $u_{0}+A^{\perp}$, they will have to converge towards the unique minimizer $u_{0}+\bar{u}$.

Remark 6 Despite the strong convergence in this statement, it may not be true that the error is coercive, even restricted to $u_{0}+A^{\perp}$, so that strong convergence could be very slow. Because of this same reason, it may be impossible to establish rates of convergence for these minimizing sequences.

The element $u_{0}$ determines the non-homogeneous data set of each problem: source term, boundary conditions, initial and/or final condition, etc. The subspace $H$ is the subset of the ambient Hilbert space $X$ for which the data set vanishes. $\mathbf{T}$ is the operator defining the corrector, so that Ker $\mathbf{T}$ is the subspace of all solutions of the underlying equation or system. The subspace $A$ is the subspace of all solutions of the problem with vanishing data set. The important property is (iii) in the statement guaranteeing that we indeed have strong convergence in $X$ of iterates. The main requirement for this to hold is to know, $a$ priori, that the error attains its minimum value zero somewhere, which in the situation treated here is guaranteed by Theorem 1.

Proof of Proposition 3.1- The result is obtained by applying Lemma 3.1 as follows. If we put $B=\left\{\mathbf{y} \in \mathbf{L}^{2}\left(0, T, \mathbf{H}_{0}^{1}(\Omega)\right): \mathbf{y}_{t} \in \mathbf{L}^{2}\left(0, T ; \mathbf{H}^{-1}(\Omega)\right)\right\}, X$ is taken to be $B \times L^{2}(0, T ; U) \times \mathbf{L}^{2}\left(q_{T}\right)$. $H$ is taken to be $\mathcal{A}_{0}$ as given in (9) and $u_{0}=s_{\mathbf{0}} \in \mathcal{A} \subset X$. The operator $\mathbf{T}$ maps a triplet $(\mathbf{y}, \pi, \mathbf{f}) \in \mathcal{A} \subset X$ into the corresponding corrector $\mathbf{v} \in Y=\mathbf{H}_{0}^{1}(\Omega)$ as explained earlier.

Remark 7 The construction of the minimizing sequence only requires the resolution of standard well-posed elliptic problems over $Q_{T}$, well-adapted to general situations (time dependent support, mesh adaptation, etc). On the other hand, it is important to highlight that the $\mathbf{L}^{2}\left(q_{T}\right)$ control function $\mathbf{f}$ obtained from the minimizing procedure does a priori not minimize any particular norm (for instance the $\mathbf{L}^{2}-$ norm).

We refer to [10] for more details and numerical illustrations of our approach in the 2D dimensional case.

\section{References}

[1] B. Bochev, M. Gunzburger, Least-squares finite element methods., Applied Mathematical Sciences, 166. Springer, New York, 2009. xxii+660 pp.

[2] J-M. Coron, Control and nonlinearity, Mathematical surveys and monographs, 136. American Mathematical Society, Providence, RI, 2007. xiv+426 pp.

[3] C. Fabre, G. Lebeau, Prolongement unique des solutions de l'équation de Stokes. (French), Comm. in PDE, 21 1996, 573-596.

[4] E. Fernández-Cara, S. Guerrero, O.Yu. Imanuvilov, J.-P. Puel, Local exact controllability of the Navier-Stokes system, J. Math. Pures Appl., 83 (12)(2004), 1501-1542.

[5] A.V. Fursikov and O. Yu. Imanuvilov, Controllability of Evolution Equations, Lecture Notes Series, number 34. Seoul National University, Korea, (1996) 1-163.

[6] R. Glowinski, J.-L. Lions and J. He, Exact and approximate controllability for distributed systems: a numerical approach, Encyclopedia of Mathematics and its applications 117 Cambridge, 2008.

[7] I. Lasiecka and R.Triggiani, Control theory for partial differential equations: continuous and approximation theories. I. Abstract parabolic systems. Encyclopedia of Mathematics and its Applications, 74. Cambridge University Press, Cambridge, 2000.

[8] A. Münch and E. Zuazua, Numerical approximation of null controls for the heat equation: ill-posedness and remedies, Inverse Problems, 26(8), 39pp, (2010).

[9] A. Münch and P. Pedregal, Numerical null controllability of the heat equation through a variational approach. Preprint.

[10] A. Münch and P. Pedregal, Numerical approximations of null controls for the stokes through a least-squares appproach. In preparation.

[11] P. Pedregal, A variational perspective on controllability, Inverse Problems, 26(2010), 015004 17p.

[12] R. Temam, Navier-Stokes equations. Theory and numerical analysis. Reprint of the 1984 edition. AMS Chelsea Publishing, Providence, RI, 2001. xiv+408 pp. 